\title{
A paradigm shift is necessary to relate running injury risk and footwear design - comment on Nigg et al.
}

\author{
Joseph Hamill, ${ }^{1, *}$, Katherine A. Boyer ${ }^{1} \&$ Gillian Weir ${ }^{1}$ \\ 1 Biomechanics Laboratory, Department of Kinesiology, University of Massachusetts Amherst, USA \\ * Corresponding author: Biomechanics Laboratory, Department of Kinesiology, University of Massachusetts Amherst, 30 Eastman Lane, \\ Amherst, MA 01003, USA, Tel: +1 413-545-2245, Fax:+1 413-545-2906 \\ Email: jhamill@kin.umass.edu
}

\section{COMMENTARY}

\section{Article History:}

Submitted $13^{\text {th }}$ December 2017

Accepted $2^{\text {nd }}$ January 2018

Published $18^{\text {th }}$ April 2018

Handling Editor:

Markus Tilp

Karl-Franzens-University Graz, Austria

Editor-in-Chief:

Martin Kopp

University of Innsbruck, Austria

\begin{abstract}
In this commentary, we respond to suggestions that new paradigms are needed to relate runningrelated injury risk and footwear design. We concur with the authors of this paper that the previous paradigms on which footwear were designed are faulty. We also concur with the authors that new paradigms are indeed needed and that research must take into consideration more epidemiological studies and more prospective biomechanical studies. The authors suggest new paradigms including muscle tuning, the preferred movement path and functional groups. However, we do raise questions about each of these suggestions regarding how these paradigms can be developed in future research designs.
\end{abstract}

Keywords:

injury risk - ground reaction forces - pronation - muscle tuning - preferred motion path

\section{Citation:}

Hamill, J., Boyer, K. A. \& Weir, G (2018): A paradigm shift is necessary to relate running injury risk and footwear design - comment on Nigg et al. Current Issues in Sport Science, 3:104. doi: 10.15203/CISS_2018.104

This is a commentary on a CISS report article authored by Nigg, B. M., Mohr, M. \& Nigg, S. R. (2017). Muscle tuning and preferred movement path - a paradigm shift. Current Issues in Sport Science, 2:007. doi: 10.15203/CISS_2017.007

\section{Introduction}

The popularity of running as a form of physical activity began in the 1970's instigating the development of sports shoes designed from a biomechanical perspective. At this time, the biomechanical considerations of running footwear focused on two guiding principles: 1) decreasing the risk of running related injuries; and 2) improving performance. For the most part, the former has taken precedence over the latter. There has been two injury-related foci that have been studied extensively: 1) cushioning the shoe during foot/ground collisions; and 2) controlling rearfoot calcaneal eversion/pronation. However, despite advancements in research and subsequent improvements in running footwear design over the years, the rates of running-related injuries have not decreased (Taunton et al., 2002). While footwear is an injury risk factor, it must be considered along with several other risk factors. Overall, there certainly is a need for new paradigms to form the basis for footwear design.

\section{Reducing the Load During the Foot/ Ground Collision}

The relationship between impact forces and injury was derived initially from animal studies in which joints were subjected to numerous repeated impacts (Radin \& Paul, 1971). In this study, bovine joints were significantly degraded suggesting that the repeated impacts were deleterious and a risk factor for injury. However, several studies have provided evidence suggesting that high impact loading is not necessarily linked to running injuries. Such studies have reported that knee osteoarthritis is found in equal frequency in runners and non-runners (e.g. Lane et al., 1986). Nigg (2001) reported that runners with a higher 
injury frequency actually had lower maximum vertical impact force and loading rate than those who had higher values of these parameters. Thus, it appears that, unlike previous thinking, neither high impact peaks nor high loading rates relate directly to injury. As these authors note, the use of ground reaction forces (GRF) (i.e. initial vertical peak and vertical loading rate) is not the most appropriate method for evaluating impact load. However, in the biomechanics literature, there are still a number of studies supporting a relationship between higher vertical GRF parameters and injury (e.g. Milner et al., 2006).

\section{Rearfoot Pronation/Calcenal Eversion}

Possibly the most misleading concept in footwear design is the pronation paradigm. Pronation (or its surrogate, calcaneal eversion) is a natural foot motion. This natural motion has been proposed to be related to the preponderance of knee injuries by the linkage of calcaneal eversion, tibial internal rotation and knee flexion. It has been suggested that if there is excessive calcaneal eversion or calcaneal eversion occurs late in the stance phase, greater stress would be placed on the knee joint with tibial internal rotation lasting well into the knee extension phase of support. The term 'excessive-' or 'over-pronation' thus was thought to be a risk factor for injury and footwear manufacturers created footwear to reduce or minimize pronation. However, there is no clinical definition for 'normal' pronation so it is difficult to determine what is excessive. Thus, the cause of 'pronation like' injuries is speculative at best. Possibly evaluating eversion using a 'pronation buffer' paradigm (Rodrigues et al., 2015) may shed more light on the use of eversion as a design feature in footwear.

As Nigg and associates (2017) point out, there are a number of methodological considerations that must be considered in measuring pronation. First, markers placed on the shoe do not reflect the movement of the foot, presenting a critical measurement issue. Next, pronation should be measured about the sub-talar joint axis that is an oblique axis and results in pronation being very difficult to measure. The surrogate measure commonly used for pronation is calcaneal eversion that is commonly determined as a rotation about the long axis of the foot. Lastly, the inter-subject variability is extremely large. Thus, the parameters derived from the calcaneal eversion angle have not been strongly correlated with injury risk (e.g. Nielsen et al., 2014).

\section{Solution to Footwear Design}

Muscle Tuning - The authors' proposed concept of muscle tuning suggests that there is a neuromuscular response at or prior to foot contact in running that alters the damping properties of the soft tissue compartments. This limits both the magnitude and number of cycles of soft tissue compartment vibration. The proposed concept of muscle tuning hinges on the idea that im- pact forces must be important in large part because people can perceive differences in them but not for the initially proposed reasons of impact force magnitude related injuries. Prior to the concept of muscle tuning, wobbling mass models (e.g. Cole et al., 1995) of the impact phase in running have been used to understand the determinants of impact force characteristics. These types of models have also shown that soft tissue compartment motion plays an important role in the dissipation of energy upon landing (Pain \& Challis, 2002). However, what is not yet known, but critical for understanding the potential importance of designing footwear or apparel for the muscle tuning concept, is the consequences of failing to tune the soft tissue compartments appropriately for landing.

A conceptual framework of the biological relevance for minimizing the soft tissue compartment vibration in running, particularly in the proposed cases where resonance is possible (fast running and low muscle tonus), is needed. As demonstrated by the study using a vibration platform (Wakeling et al., 2002), the body can and does respond to a continuous vibration stimulus. The relevance of this response and negative effects of continuous vibration to the human body is well established from studies of workplace performance and injury. In response to repetitive impacts, anecdotal evidence of injuries in circus athletes due to a non-uniform surface suggests that it is the unexpected landing situation that matters most. Thus, it may follow that the muscle tuning that occurs during running is likely small and may not be relevant for recreational runners. However, in high performance runners, where the risk of resonance may be higher due to faster stride rates and small improvements in efficiency are valuable, the additional muscle activity needed to properly tune the soft tissue compartment may be detrimental to performance. As suggested by the authors, quantifying the muscle tuning response is extremely challenging due to the close coupling of a muscle response to damping vibrations with a muscle response to alter the limb position for landing. While the authors imply that these may be two separate things, the possibility that both: 1) changes in damping; and 2) controlling the impact phase by altering limb geometry, are motivated by a need to minimize soft tissue compartment vibrations should be considered together.

The Preferred Movement Path - In several previous studies (e.g. Nigg, 2001), Nigg suggested that there is a subject and task specific locomotion pattern that is determined by multiple factors (i.e. muscles, tendon, ligaments, bone structure, etc.). He further suggested that this particular motion path may explain why shoes have little or only a moderate effect on lower extremity kinematics (Stacoff et al., 2000). The authors of the current paper suggest that footwear should be designed to facilitate the individual's preferred movement path and that such footwear designs could be energetically advantageous. This is an intriguing hypothesis and, for all practical purposes, makes a great deal of sense. The observed inter-runner variability that results in a mean possibly not resembling any individual in the group may be a result of the individual preferred paths of each of the runners. The major challenge may be determining the in- 
dividual's preferred locomotor pattern. One problem concerns the possibility that an individual's movement path can change from one day to another or possibly as a function of fatigue. Thus, we have to ask what is the runner's preferred movement path, does an individual have multiple preferred motion paths (i.e. dependent upon fatigue), and how can we determine that/ those path/paths?

Functional Groups - Certainly, there may be a group of individuals who respond to a specific shoe intervention in a similar way. The authors have suggested that these individuals form a'functional group'. This concept is not new; footwear manufacturers have already determined functional groups for those who need cushioning, stability or motion control footwear. However, this grouping is based on old paradigms (i.e. impact loading and pronation). The development of new functional groups requires sound, well-researched paradigms as the authors point out. However, taken to the extreme, a functional group could be a single individual and ultimately we could customize footwear for a single individual.

\section{Conclusion}

Many of the implications of previous studies relating footwear and injury risk were derived from extremely small samples thus lacking sufficient statistical power to detect true differences. In addition, many of these studies were retrospective in nature. It is clear that prospective and/or epidemiological studies with large sample sizes are a necessity to discern the link between footwear and injury risk. Large-scale studies (e.g. Nielsen et al, 2014) indicate that the basis for the design of running footwear may have been misguided. It is clear that new paradigms for relating biomechanically informed footwear design and injury risk are necessary.

\section{Funding}

The authors have no funding or support to report.

\section{Competing Interests}

The authors have declared that no competing interests exist.

\section{References}

Lane, N.E., Bloch, D.A., Jones, H.H., Marshall, W.H., Wood, P.D., \& Fries, J.F. (1986). Lone distance running, bone density and osteoarthritis. Journal of the American Medical Association, 255 (9), 1147-1151.

Milner, C.E., Ferber, R., Pollard, C.D., Hamill, J., \& Davis, I.S. (2006). Biomechanical Factors Associated with Tibial Stress Fracture in Female Runners. Medicine \& Science in Sports \& Exercise, 38 (2), 323-328.

Nielsen, R.O., Buist, I., Palmer, E.T., Nohr, E.A., Sørensen, H., Lind, M., et al. (2014). Foot pronation is not associated with increased injury risk in novice runners wearing a neutral shoe: a 1-year prospective cohort study. British Journal of Sports Medicine, 48 (6), 440-447.

Nigg, B.M. (2001). The role of impact forces and foot pronation: a new paradigm. Clinical Journal of Sport Medicine, 11 (1), 2-9.

Nigg, B.M., Mohr, M.M., \& Nigg, S.R. (2017). Muscle tuning and preferred movement path - a paradigm shift. Current Issues in Sport Science, 2:007. doi: 10.15203/CISS_2017.007.

Pain, M.T., \& Challis, J.H. (2002). Soft tissue motion during impacts: their potential contributions to energy dissipation. Journal of Applied Biomechanics, 18 (3), 231-242.

Radin, E.L., \& Paul I.L. (1971). Response of joints to impact loading. Arthritis and Rheumatism, 14 (3), 356-362.

Rodrigues, P., Tenbroek, T., \& Hamill, J. (2013). Runners with anterior knee pain use a greater percentage of their available pronation range of motion. Journal of Applied Biomechanics, 29, 141-146.

Stacoff, A., Nigg, B.M., Reinschmidt, C., van den Bogert, A.J., \& Lundberg, A. (2000). Tibiocalcaneal kinematics of barefoot versus shod running. Journal of Biomechanics, 33 (11), 1387-1395.

Taunton, J., Ryan, M.B., Clement, D.B., McKenzie, D.C., LloydSmith, D.R., \& Zumbo, B.D. (2002). A retrospective casecontrol analysis of 2002 running injuries. British Journal of Sports Medicine, 36:95-101.

Wakeling, J.M., Nigg, B.M., \& Rozitis, A.I. (2002). Muscle activity damps the soft tissue resonance that occurs in response to pulsed and continuous vibrations. Journal of Applied Physiology, 93(3), 1093-1103.

\section{Data Availability Statement}

All relevant data are within the paper. 